\title{
Emulating Sentinel-1 Doppler Radial Ice Drift Measurements using Envisat ASAR Data
}

\author{
Thomas Kræmer, Harald Johnsen, Camilla Brekke
}

\begin{abstract}
Using data from the Envisat ASAR instrument, this paper demonstrates how the high precision radial surface velocity product, which will become available with ESA's Sentinel-1 satellite, can complement the analysis of sea ice motion. Highresolution Doppler frequency measurements are used to estimate the sub-second line-of-sight motion of drifting sea ice in Fram Strait. We compare the method with buoy measurements as well as a recent cross-correlation algorithm for tracking ice between pairs of images. Maximum speeds measured from the time series were in the order of $20 \mathrm{~cm} / \mathrm{s}$. Using our method we measured instantaneous speeds reaching $40-60 \mathrm{~cm} / \mathrm{s}$.
\end{abstract}

Index Terms-Sea ice, motion estimation, synthetic aperture radar, Doppler measurements, Sentinel-1

\section{INTRODUCTION}

The motion and deformation of sea ice has a major impact on the ice thickness distribution. Diverging ice creates leads where new ice can grow, and converging ice piles up and forms ridges. On a pan-Arctic scale, information on sea ice motion is needed to quantify ice volume exchanges and for understanding the momentum, mass and energy balance of the Arctic Ocean (see figure 1 for an overview of large scale ice drift patterns in the Arctic).

On a local scale, tracking of potentially dangerous ice such as icebergs, multi-year ice and ridges is required to prevent damages to ships as well as oil and gas installations in the Arctic Ocean. The detection of opening leads also allows for faster navigation through ice infested waters.

Sea ice motion has traditionally been estimated from time series of satellite images using both optical and microwave sensors. Algorithms find common patterns in pairs of images by use of multi-scale cross-correlation between small image blocks or by matching derived features such as boundary polygons of floes or leads (see [1] and references therein). The matched coordinates together with the time difference between acquisitions provide estimates of the ice displacement and velocity. Active microwave sensors are by far the most popular due to their ability to sense independent of daylight and cloud cover. In particular, synthetic aperture radar (SAR) provides a nice balance between wide coverage and good spatial resolution.

In contrast to the high spatial resolution, the temporal resolution of satellite SAR time series is low relative to certain weather events. During the 1-3 days separating two

T. Kræmer and C. Brekke are with the Department of Physics and Technology, UiT - The Arctic University of Norway, 9037 Troms $\varnothing$, Norway (e-mail: thomas.kramer@uit.no).

H. Johnsen is with the Northern Research Institute (NORUT).

The work was supported by the Arctic Earth Observation and Surveillance Technologies (Arctic EO) project 195143 under contract of the Norwegian Research Council. acquisitions, wind and currents may have rendered patterns in the ice untraceable, especially in the highly dynamic marginal ice zone (MIZ). This decorrelation may cause large gaps in the estimated flow fields. Furthermore, the estimated flow fields only provide average velocities. It has been found that traditional tracking of ice drift from image time series leads to a consistent underestimation of the true drift speeds with biases reported to be as large as 10\%-20\% when tracking floes from images separated by 1-3 days [2]. The underestimation is a consequence of the fact that correlation methods connect matched points by straight lines whereas the real path is likely more complex. More precisely, the speed is calculated based on the displacement of the ice rather than the traveled distance.

Higher time resolution is usually obtained by use of buoys on the ice or upward looking sonar. These instruments are valuable, but they only provide point measurements. This motivates the search for methods which will allow us to observe near real-time ice speeds from satellite over an extended area.

In this paper we use Doppler frequency measurements from the Envisat Advanced Synthetic Aperture Radar (ASAR) instrument to estimate the radial surface velocity of the drifting sea ice over an extended area. Sections II and III define the relevant quantities and present their estimation and proper calibration. With the launch of Sentinel-1 these measurements will become routinely available; our study illustrates the possibilities of using such products in sea ice research. Our results are compared to the output from a recent cross-correlation method as well as measurements from a drifting ice buoy (section IV). We use examples from the Fram Strait, which is an interesting area due to higher ice speeds compared to the central Arctic [3].

\section{RADIAL SURFACE VELOCITY FROM SAR}

By radial surface velocity we mean the ground range component of the motion of scatterers on the Earth's surface, parallel to the antenna pointing direction. During some satellite passes, the antenna pointing in the range direction aligns with the motion of the sea ice. Two techniques allow us to directly measure radial surface velocities from SAR data: (i) alongtrack interferometry (ATI) which requires a second receiving antenna and (ii) lower resolution single-antenna Doppler shift measurements. Although the resolution of ATI is impressive under optimal baseline conditions $(33 \mathrm{~m} \times 33 \mathrm{~m}$, see [4]), such products will not be routinely available in the near future. ATI also requires a high degree of coherence between scenes, which is not always the case as sea ice is a highly dynamic medium. During early ice formation, the temporal decorrelation time will be close to that of open sea surfaces 


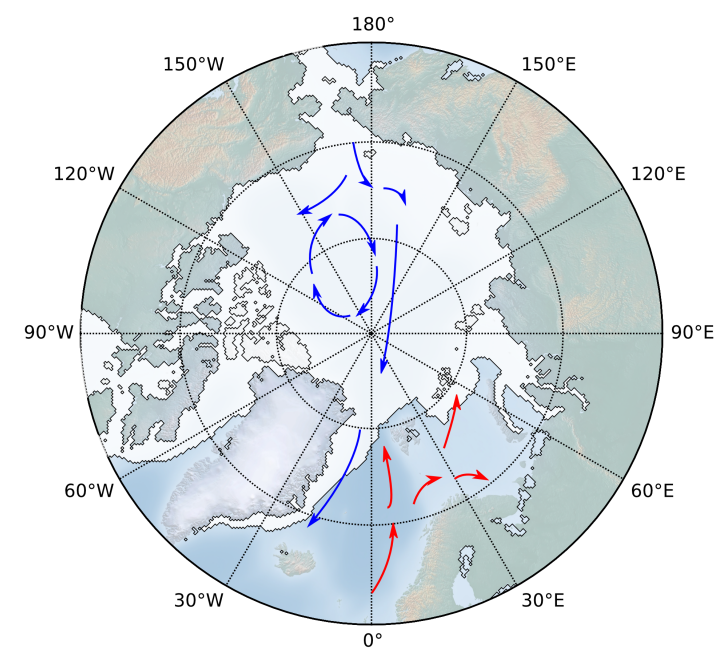

Fig. 1. Mean ice drift (blue arrows) and ocean current patterns (red arrows). The blue arrows in the center show the Beauford gyre and the transpolar drift. Adapted from [5, fig. 3.1(a)].

which is in the order of $50 \mathrm{~ms}$ at C-band, while deformation monitoring of fast ice can be conducted with a temporal baseline of hours.

The single-antenna Doppler frequency measurements, however, have been available with the Envisat ASAR wide swath mode (WSM) product since 2007 and will continue to be made available through the Sentinel-1 Level-2 ocean (OCN) product [6]. Each OCN product contains up to three geophysical components: the radial surface velocity (RVL), the ocean surface wind field (OWI) and the ocean swell wave spectra (OSW) components. In this paper we show the potential of the RVL product for sea ice drift estimation.

The body of literature concerning the single-antenna Doppler method for estimating the radial surface velocity for ocean wind and current retrieval is growing [7]-[9]. However, use of Doppler velocity measurements for sea ice applications is still in its infancy. Fujiyoshi et al. (2013) experimented using ground based 3D scanning X-band Doppler radar to estimate ice velocities from the coast near Sea of Okhotsk [10]. To the authors' knowledge, the only published study on sea ice drift using single-antenna spaceborne Doppler shift measurements was by Hansen et al. [11] using the Doppler grid available with the ASAR WSM product. The resolution of the ASAR WSM Doppler grid is relatively low $(\sim 4 \mathrm{~km} \times$ $8 \mathrm{~km}$ in range and azimuth respectively) because the Doppler centroids are estimated directly from the raw SAR data which requires substantial averaging to obtain the required precision.

In this paper we use the estimation strategy of the Sentinel1 RVL product where Doppler frequencies are estimated from single-look complex (SLC) data. For the Sentinel-1 interferometric wide-swath (IW) mode this provides a higher resolution grid with $\sim 1 \mathrm{~km} \times 1 \mathrm{~km}$ cells with an effective resolution of $\sim 2 \mathrm{~km} \times 2 \mathrm{~km}$ mid-swath [12].

\section{ESTIMATION METHODOLOGY}

This section provides an overview of the process for obtaining calibrated radial surface velocities from observed Doppler frequency measurements (see figure 2). The output is a normalized radar cross section (NRCS) image, a bias-corrected Doppler frequency image which can be converted to an RVL image and the predicted standard deviation of the Doppler estimates (STD). The quantities discussed are expressed in standard SI units where $f$ denotes a linear frequency in $\mathrm{Hz}$, $t$ denotes time in seconds, $c$ denotes a speed in meters per second and $\lambda$ denotes a wavelength in meters. For exact details of the Doppler estimation process, we refer the reader to the RVL algorithm specification document [13].

\section{A. Doppler centroid estimation}

The Doppler centroid, $f_{\mathrm{Dc}}$, defined as the radar return frequency shift at the antenna beam center, is related to the relative motion between the satellite platform and the rotating Earth

$$
f_{\mathrm{Dc}}=-2 \frac{v_{\text {rel }}}{\lambda}
$$

where $v_{\text {rel }}$ is the effective relative velocity between the SAR instrument and the Earth surface and $\lambda$ is the carrier wavelength of the SAR system [14]. The sign convention is such that the Doppler frequency is positive for scattering elements approaching the radar and negative for elements moving away from the radar. Precise estimation and calibration of $f_{\mathrm{Dc}}$ forms the basis of estimating radial surface velocities.

The starting point for the Doppler centroid estimation is raw (unfocused) Level-0 Envisat ASAR image mode (stripmap) data. Each scene was focused to an SLC image using the full bandwidth of the data, i.e. without applying any window functions. The Doppler centroid is then estimated from SLC data using the methodology of Bamler [15] extended to compensate for side band effects [13]. By side band effects we mean aliasing of energy from the side-lobes of the antenna pattern into the main lobe due to strong intensity gradients in the azimuth direction. This often happens close to land, where the topography may cause bright returns, but any large change in backscatter intensity may affect the estimation. Hansen et al. [16] corrected for this effect by post-processing of the estimated Doppler frequencies based on a linear fit between the Doppler frequencies and the backscatter intensity. In our method, this correction is done directly as part of the Doppler estimator, using the method presented in [12], [13]. The Doppler centroid estimation is performed block-wise using 992 azimuth lines and 192 range pixels with overlap steps of $1 / 4$ side length (in both directions), producing a $\sim 1$ $\mathrm{km} \times 1 \mathrm{~km}$ grid with a resolution of $\sim 2 \mathrm{~km} \times 2 \mathrm{~km}$ mid-swath.

The stable orbit and attitude of the Envisat satellite allows us to accurately predict the contribution to the Doppler centroid due to the satellite-Earth geometry. Chapron et al. [9] showed that Doppler centroid measurements do not agree perfectly with the predicted Doppler centroid and that the anomaly can be related to a geophysical movement of scatterers on the Earth's surface. The quantity of interest is therefore this residual motion. We define the Doppler centroid anomaly as

$$
f_{\text {Dca }}=f_{\text {Dc }}-f_{\text {geom }}
$$

where $f_{\mathrm{Dc}}$ is the observed Doppler centroid and $f_{\text {geom }}$ is the predicted geometric Doppler shift due to the moving Earth 


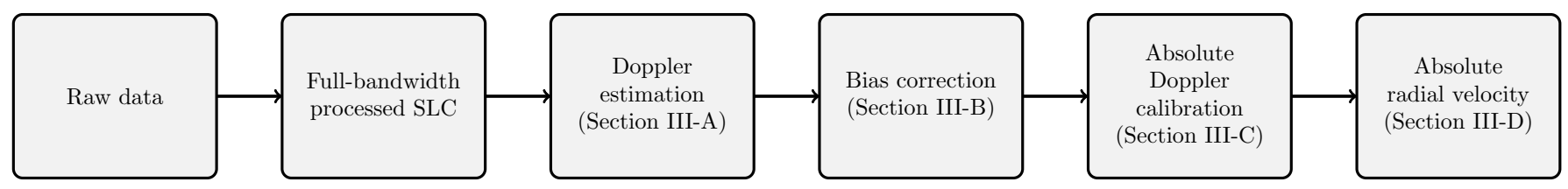

Fig. 2. A schematic overview of the estimation and calibration process for the RVL product.

[14]. To calculate $f_{\text {geom }}$ we need the orbit, attitude and relative velocity between sensor and a target on the surface at the beam center, which is readily found by solving the Range-Doppler equations using precise state vectors [14], [17]. A geophysical Doppler shift is then related to the Doppler centroid anomaly by

$$
f_{\text {phys }}=f_{\text {Dca }}-f_{\text {bias }}
$$

where $f_{\text {bias }}$ is the total error due to uncertainties in the orbit, attitude, antenna pattern and topography. After correcting known biases, $f_{\text {phys }}$ can be converted to a radial surface velocity, as described in section III-D. The following section discusses systematic biases contributing to $f_{\text {bias }}$ and how to correct them.

\section{B. Bias corrections}

In this section we discuss the dominant biases in the Doppler anomaly that should be corrected before interpretation of the results. For calibration purposes it is customary to use images containing large areas of homogeneous backscatter. Figure 3a shows the estimated $f_{\text {Dca }}$ for a VV polarization scene acquired over the Borneo rainforest 24 July 2004 in the IS5 swath. Over a rainforest scene we expect the Doppler anomaly to be zero over homogeneous areas, but in figure 3a we can easily identify two biases that need to be corrected to properly calibrate the Doppler measurements: a slow varying trend in the range direction and a rapid periodic variation in the azimuth direction. We assume that the biases are independent and can be corrected for separately.

1) Range bias: In the range direction, the pattern due to electronic mispointing of the antenna is clearly visible. The bias, denoted $f_{\text {bias }}^{\text {range }}(x)$, varies with range cell $x$ and is a result of the degradation of the transmit/receive modules of the ASAR antenna array over time [18]. This is a slowly time-changing bias which varies with polarization and swath. An estimate of the bias can be obtained by analysis of the Doppler anomaly calculated over a scene with homogeneous backscatter. Typically this is done using rainforest scenes and we use the Borneo scene as an example. However, for the sea ice images we did not have rain forest scenes which were close in time. We therefore estimated the profile over a homogeneous part of the ice where the Doppler standard deviation was less than $5 \mathrm{~Hz}$. An estimate of the electronic mispointing, which varies across the swath in the order of $45-53 \mathrm{~Hz}$ for IS5, is shown in figure $3 \mathrm{~b}$. The estimated range profile can then be subtracted from each row to correct the bias.

2) Periodic azimuth bias: In the azimuth direction, a periodic signal modulates the SLC from which we estimate the Doppler frequencies. The amplitude of this signal is small and barely visible in the intensity image $(\sim 0.1 \mathrm{~dB})$, but still large enough to severely bias the Doppler centroid anomalies. Figure 3c shows a subset of the azimuth profile obtained by averaging over all range cells in the Doppler frequency grid (figure 3a) with a standard deviation less than $5 \mathrm{~Hz}$. The profile is referred to as $\overline{f_{\text {Dca }}}$. The source of the pattern is a small difference in gain between calibration cycles in the raw data. There is a gap in acquisition every 1023rd azimuth line, where internal calibration measurements are performed. It turns out that the frequency of this periodic pattern for ASAR stripmap data is exactly half the frequency of the calibration pulses and is thus a function of the pulse repetition frequency (PRF) of the radar. The PRF varies with swath and the modulation has been found to have a period of $1.24 \mathrm{~s}$ in IS2 and $0.98 \mathrm{~s}$ in IS5.

If we take the Fourier transform $\mathcal{F}\left\{\overline{f_{\text {Dca }}}\right\}$ of the profile in figure $3 \mathrm{c}$ and plot the magnitude spectrum (see figure $3 \mathrm{~d}$ ) we see a clear peak at $0.98 \mathrm{~Hz}$ with one harmonic. For longer scenes, up to three harmonics have been observed. Note that this frequency is not a Doppler frequency, but refers to the variation of the Doppler frequency with azimuth time as shown in figure $3 \mathrm{c}$. We can estimate the parameters of a sinusoidal signal with $N$ harmonics from the magnitude and phase coefficients of $\mathcal{F}\left\{\overline{f_{\text {Dca }}}\right\}$ [19, p. 256]. Since we know the base frequency, we search a narrow band centered on the expected frequencies. The peak within each small band of the magnitude spectrum $\left|\mathcal{F}\left\{\overline{f_{\text {Dca }}}\right\}\right|$ gives an estimate of the angular frequencies $\omega_{i}$ in rad/s and amplitudes $A_{i}$. The phases $\phi_{i}$ are obtained from the corresponding position in the phase spectrum $\angle \mathcal{F}\left\{\overline{f_{\text {Dca }}}\right\}$. We then create a correction signal

$$
f_{\text {bias }}^{\text {azimuth }}(y)=\sum_{i=1}^{N} A_{i} \cos \left(\omega_{i} t_{y}+\phi_{i}\right)
$$

where $N$ is the number of harmonics and $t_{y}$ is the azimuth time corresponding to azimuth cell $y$ in the Doppler grid.

Figure $3 \mathrm{c}$ shows the estimated sinusoidal signal plotted alongside the original profile. For the scenes in this paper the periodic signal had a base amplitude $A_{1}$ in the order of 2-3 Hz. Note that the correction signal is zero-mean, but has been plotted with an offset for comparison. The zeromean sinusoidal signal is then subtracted from each column to remove the periodic bias.

\section{Absolute Doppler calibration}

After compensating for the geometric Doppler and known range and azimuth biases we expect scatterers which are not moving relative to the rotating Earth to have a Doppler centroid anomaly of zero. Due to imperfect estimation of the mentioned biases as well as inaccuracies in the predicted 


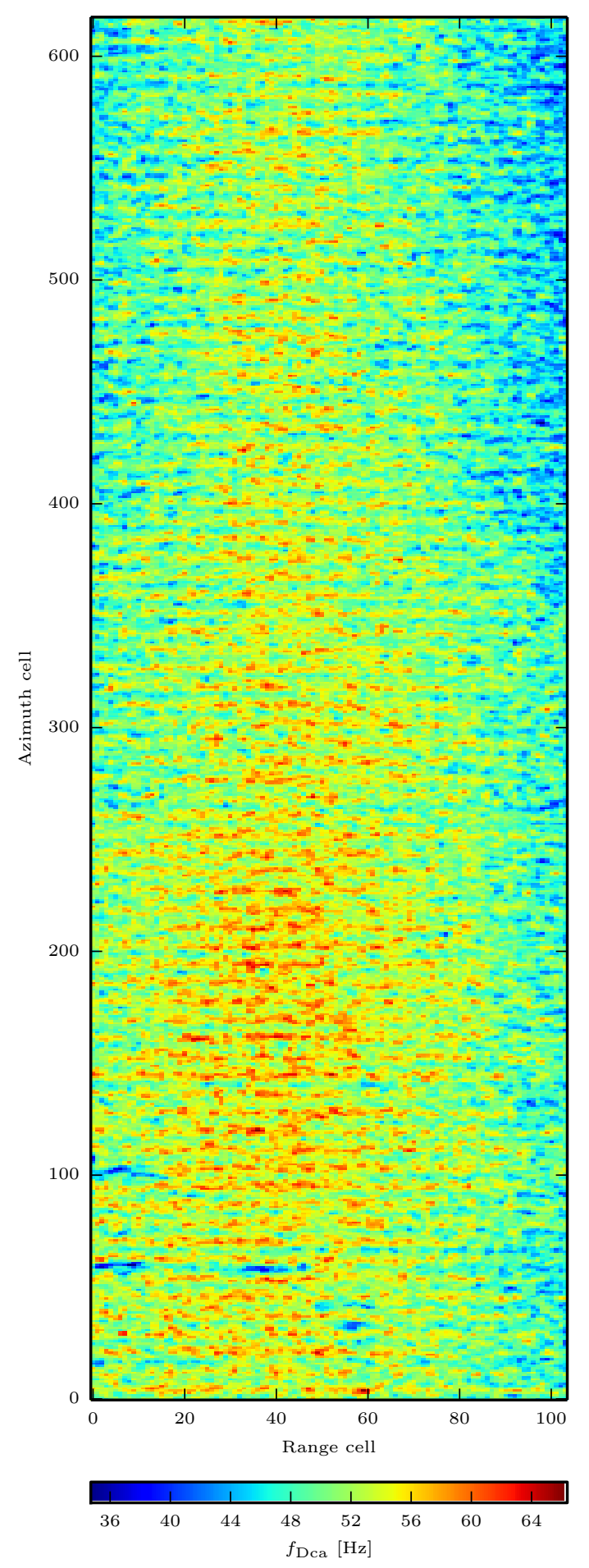

(a)

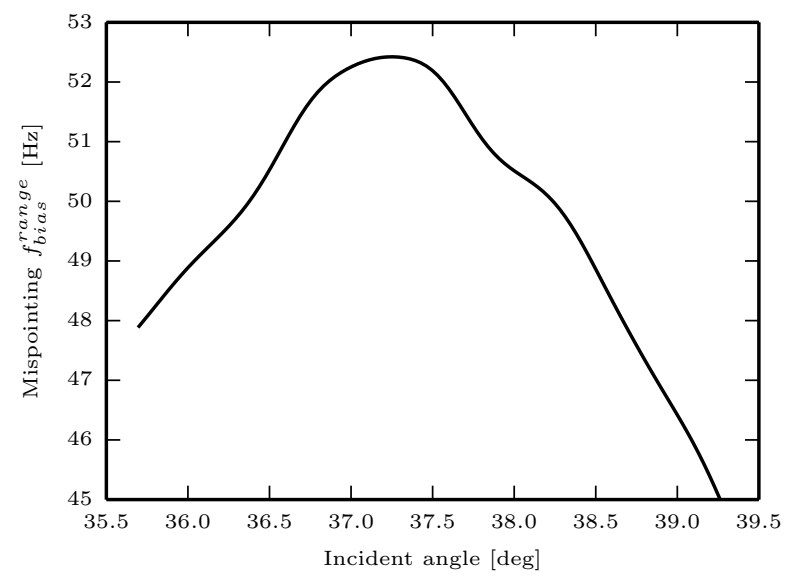

(b)

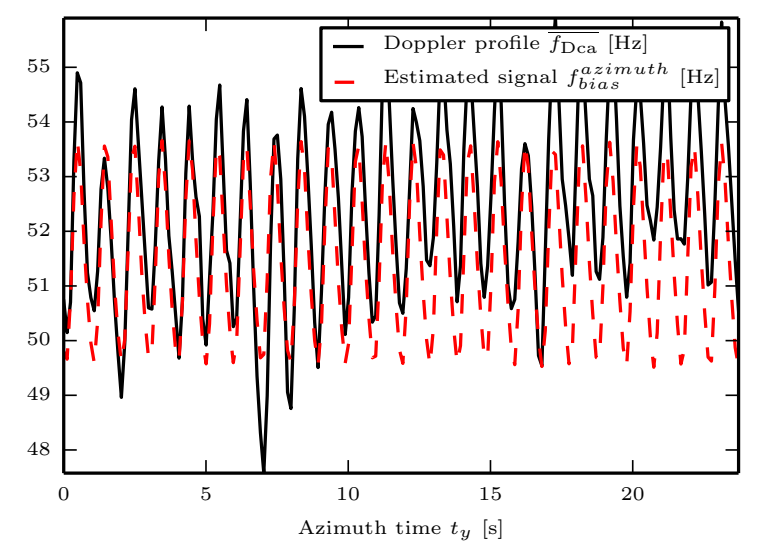

(c)

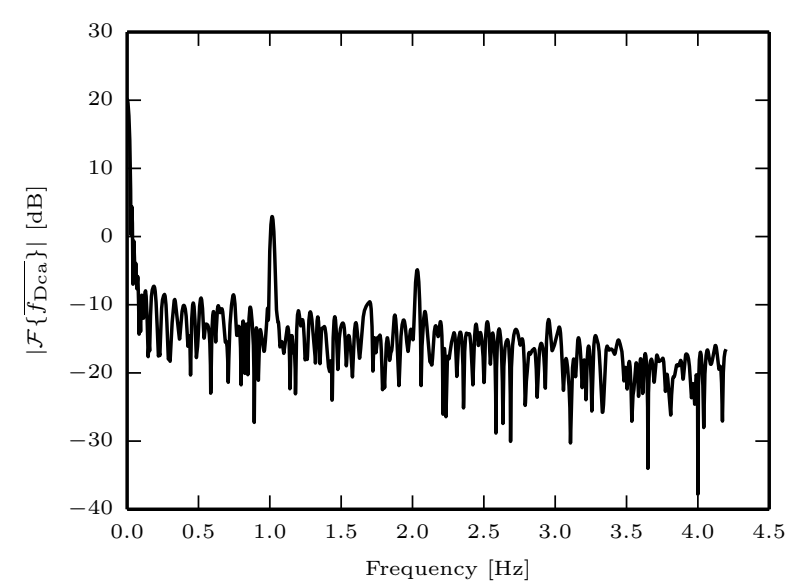

(d)

Fig. 3. Doppler frequency anomalies estimated over a rainforest scene showing the discussed biases. (a) Estimated Doppler anomaly $f_{\text {Dca. }}$ (b) Estimated electronic mispointing in the IS-5 swath as function of incident angle. (c) Mean azimuth profile of $f_{\text {Dca }}$ for lines $0-200$ showing periodic azimuth bias as well as the estimated signal. Note that the estimated signal is zero-mean, but has been given an offset for easier comparison. (d) Magnitude of the Fourier transform $\left|\mathcal{F}\left(\overline{f_{\text {Dca }}}\right)\right|$ of the profile in (c) showing two distinct peaks. 
$f_{\text {geom }}$, a residual global offset $f_{\text {bias }}^{\text {offset }}$ is sometimes observed (in the order of $1-5 \mathrm{~Hz}$ in our case). We therefore estimate this residual by averaging over land areas in the image with a land height less than $200 \mathrm{~m}$ (as in [16]) and a standard deviation less than $5 \mathrm{~Hz}$. The offset is then subtracted to produce our final estimate of $f_{\text {phys }}$. Thus, the total bias correction signal is given by

$$
f_{\text {bias }}(x, y)=f_{\text {bias }}^{\text {range }}(x)+f_{\text {bias }}^{\text {azimuth }}(y)+f_{\text {bias }}^{\text {offset }}
$$

where we have included the range cell $x$ and azimuth cell $y$ to make the independence of the range and azimuth biases explicit.

\section{Absolute radial velocity}

After correcting for known biases we can convert the geophysical Doppler values to geophysical radial surface velocities by solving equation (1) for the relative velocity and projecting to ground range

$$
v_{\text {rel }}=-\frac{\lambda f_{\text {phys }}}{2 \sin \theta_{i}}
$$

where $\theta_{i}$ is the angle of incidence. The corresponding standard deviation in $\mathrm{m} / \mathrm{s}$ is then given by

$$
\left\langle v_{\text {rel }}\right\rangle=\frac{\lambda}{2 \sin \theta_{i}}\left\langle f_{D}\right\rangle
$$

where $\left\langle f_{D}\right\rangle$ is the estimated Doppler standard deviation in Hz. The RVL grid in the Sentinel-1 product is produced from Doppler values which have been corrected for the geometric Doppler as well as antenna mispointing. However, the user will be responsible for applying any other corrections such as calibrating the measurements to land.

For easy reference, we provide a summary of the processing steps here:

- Focus the raw data to a full-bandwidth procesed SLC image (i.e. without using window functions).

- For each cell in the Doppler grid, obtain the estimated Doppler frequency $f_{\mathrm{D}}$ (eq. (30) in [13]).

- Calculate the geometric Doppler for each grid cell (see e.g. $\left[14\right.$, Chapter 12]) and subtract it from $f_{\mathrm{D}}$ to produce $f_{\mathrm{Da}}$ (equation (2)).

- Get antenna mispointing $f_{\text {bias }}^{\text {range }}(x)$ by estimating it from data or from auxiliary data and subtract it from $f_{\mathrm{Da}}$.

- Further, estimate and subtract the periodic bias in azimuth $f_{\text {bias }}^{\text {azimuth }}(y)$, then estimate and subtract any residual offset in the Doppler over land to produce $f_{\text {phys }}$.

- Convert the calibrated Doppler to a radial surface velocity using equation (6).

\section{E. Uncertainties}

We have not done a detailed investigation on the magnitude of each uncertainty. However, this section discusses sources of uncertainty which should be kept in mind when analyzing the data. We assume that the wavelength is known perfectly. Thus, the uncertainties contributing to $v_{\text {rel }}$ are those contributing to $f_{\text {phys }}$ and the incidence angle $\theta_{i}$ (see equation (6)). As discussed above, these are $f_{\mathrm{Dc}}, f_{\text {geom }}$ and $f_{\text {bias }}$.

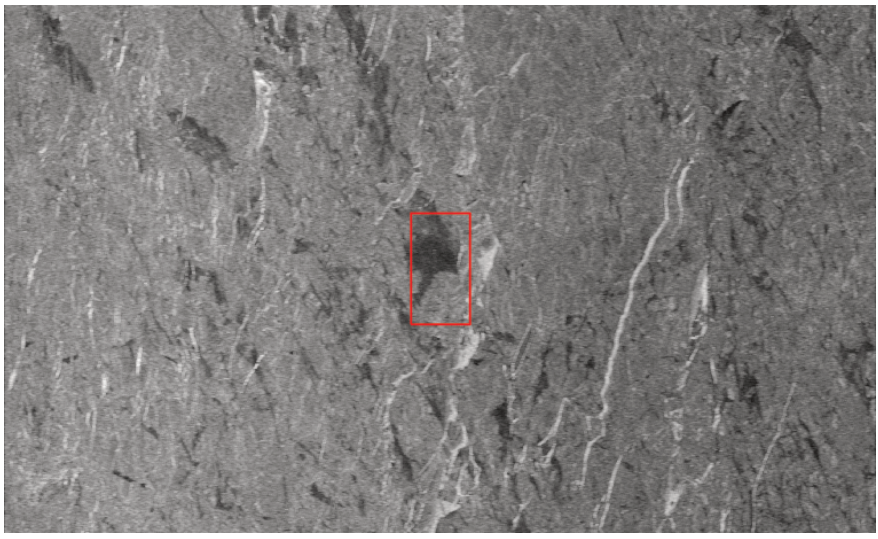

Fig. 4. Subset of an ice scene showing the size of a single Doppler estimation cell (red box) containing a mixture of ice and water. The size of the box is 992 azimuth lines and 192 range pixels $(\sim 4 \mathrm{~km} \times 3 \mathrm{~km})$. Since the estimation is done on a regular grid, the probability is high that a cell may contain both ice and water in the same cell which will increase the standard deviation of the Doppler estimates.

1) Uncertainties in $f_{D c}$ : There are two things that can influence the bias and standard deviation of the Doppler estimates $f_{\mathrm{Dc}}$ : (i) If there are strong scatterers (e.g frost flowers are known to cause strong backscatter for C-band radars [20]) close to the estimation cell, we may get aliasing into the estimation area. If side-band effects are not corrected for, this will introduce a bias and increase the standard deviation, but our approach does correct for this. (ii) There may be a mix of intensities in the SLC image and/or a mix of motions inside the physical estimation area or both. Figure 4 shows a subset of the full resolution image and a Doppler estimation cell where such mixing occurs. If there is a mix of intensities the Doppler standard deviation will increase, but we still obtain an unbiased estimate of the average Doppler centroid. There could potentially also be multiple motions inside the estimation cell. In this case we still get an unbiased estimate for the average motion inside the cell, but it may not be very informative.

2) Uncertainties in $f_{\text {geom: }}$ : The accuracy of $f_{\text {geom }}$ and the angle of incidence $\theta_{i}$ depends on the precision of the orbit state vectors and the solution of the Range-Doppler equations [14]. We have used the Doppler Orbitography and Radio-positioning Integrated by Satellite (DORIS) precise orbit state vectors which have an accuracy of $30 \mathrm{~cm}$ along each axis and the Range-Doppler equations are solved iteratively to centimeter precision [21].

3) Uncertainties in $f_{\text {bias }}$ : The total bias, $f_{\text {bias }}$, is composed of measurements of the electronic mispointing of the antenna, the periodic azimuth pattern and any residual offset. In our study with ASAR data, the largest unknown is the antenna mispointing as we estimated this over the ice sheet within each image. For Sentinel-1, the mispointing will be monitored continuously making the correction more reliable.

The periodic azimuth variation seems to be specific to the stripmap mode for both ASAR and Sentinel-1. Sentinel-1 will be using the extended wide-swath (EW) mode to study polar sea ice and the wave (WV) mode over ocean, neither of which seem to be affected by this phenomenon. 


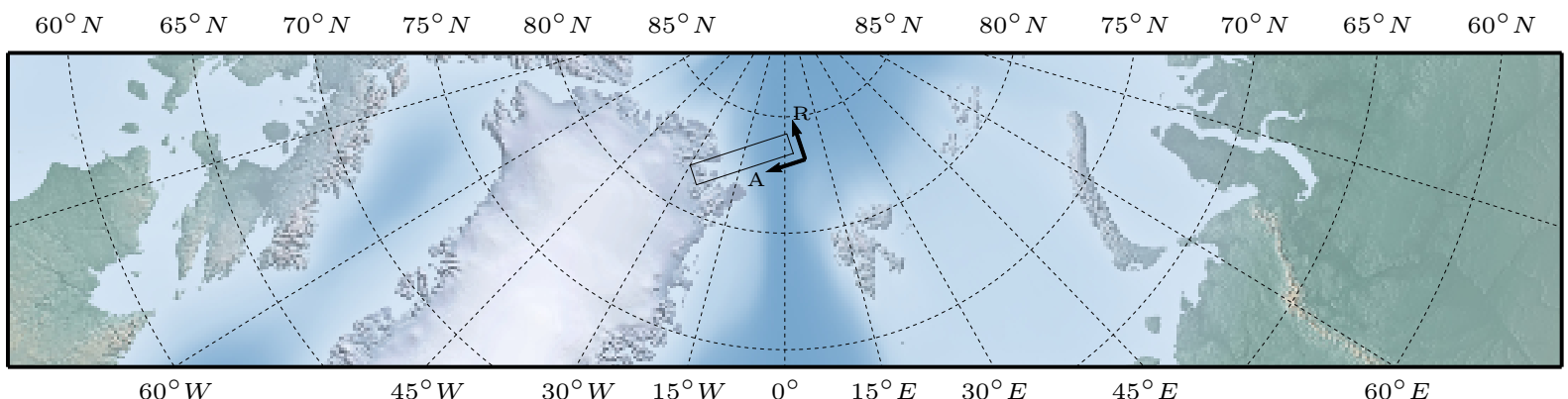

(a)

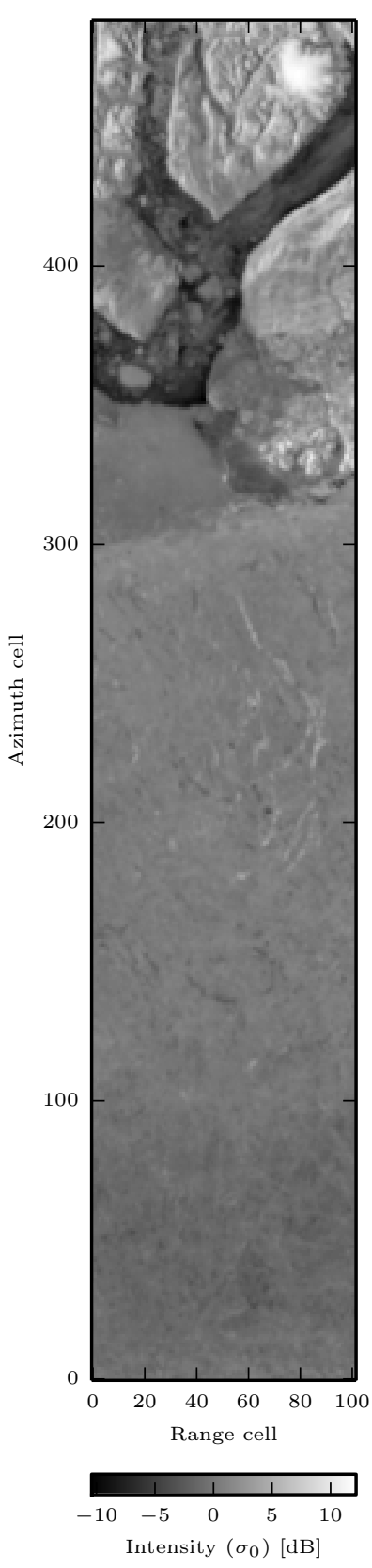

(b)

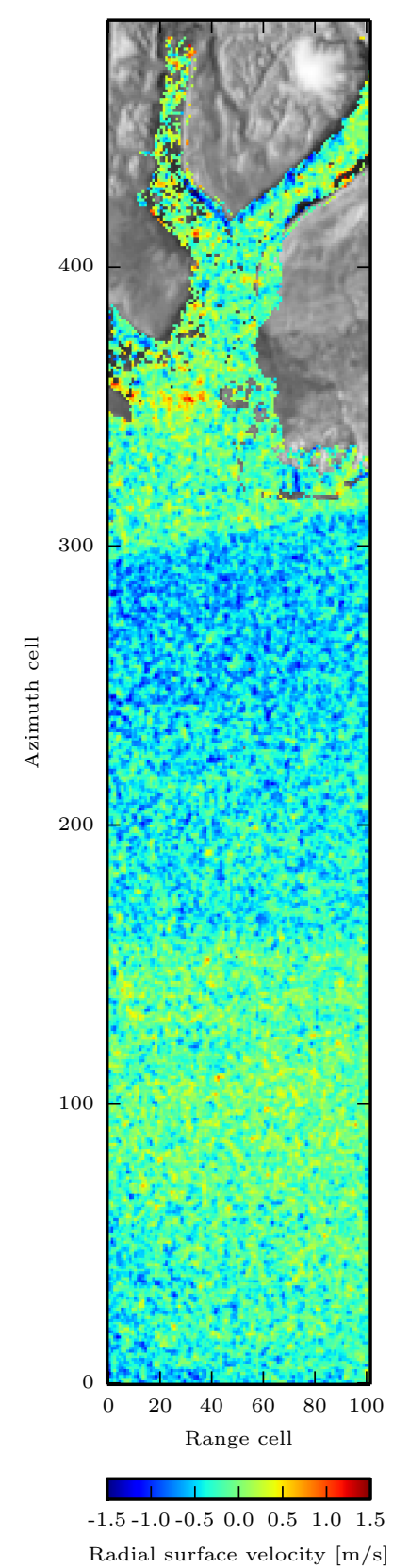

(c)

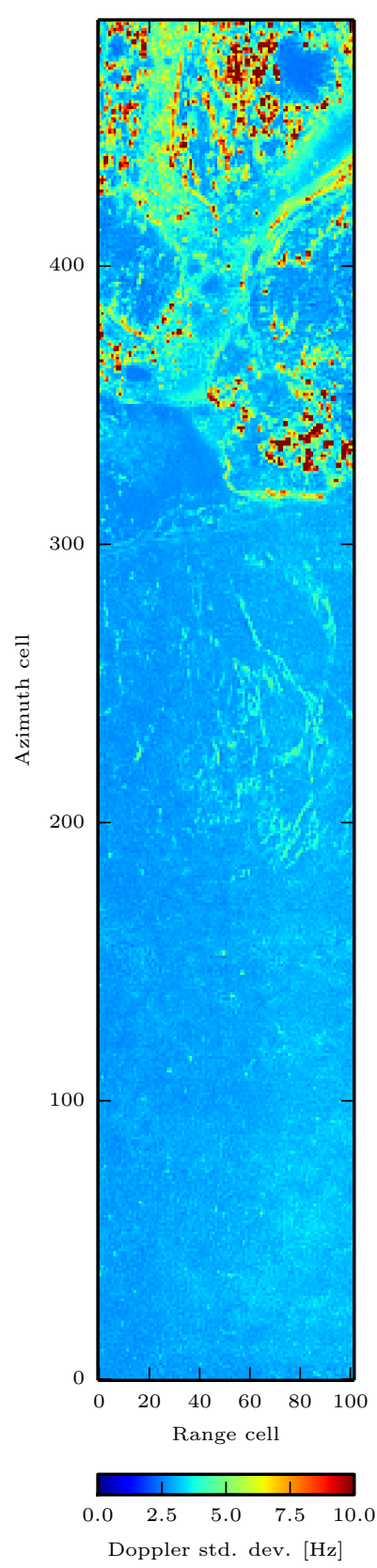

(d)

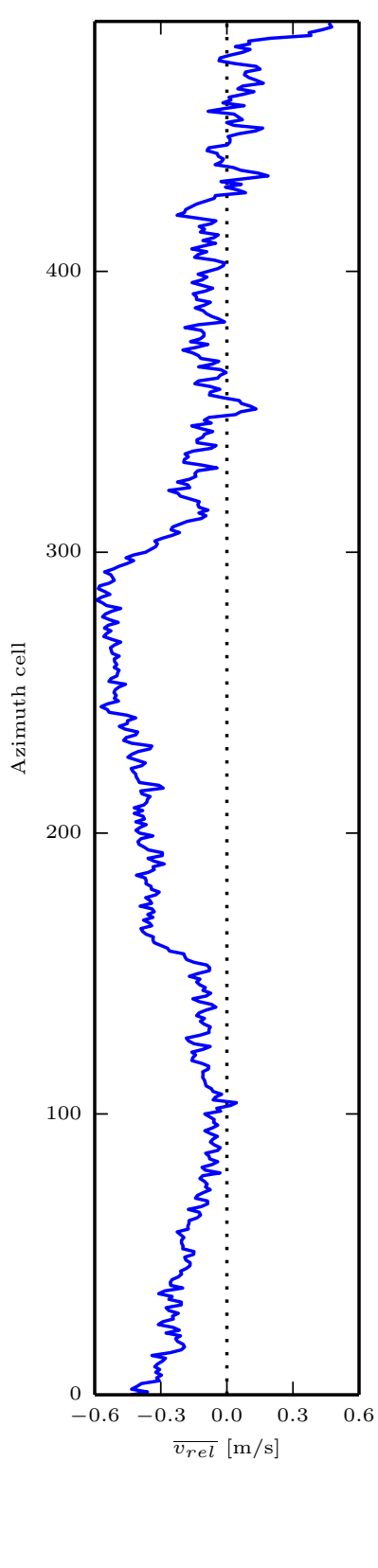

(e)

Fig. 5. Scene from 31 January 2009 (descending pass, HH polarization) showing ice moving along the Greenland coast. (a) Geographical position of the scene. The azimuth (A) and range (R) directions are indicated by arrows. (b) $\sigma_{0}$ intensity image. (c) Estimated ground range radial surface velocities showing a possible shear in the ice along the Greenland coast. (d) Standard deviations of Doppler frequency measurements. (e) Average radial velocity profile. 
TABLE I

SCENES DISCUSSED IN THIS PAPER.

\begin{tabular}{|c|c|c|c|c|c|c|c|}
\hline Date (UTC) & Orbit & Track & Swath & Pass & Polarization & Inc. angle [deg] & Content \\
\hline $24.07 .2004 \quad 02: 52: 58$ & 12539 & 461 & I5 & Ascending & VV & $35-39$ & Rainforest \\
\hline 07.11.2007 19:16:44 & 29741 & 128 & I2 & Ascending & VV & $19-26$ & Sea ice and ocean (Fram Strait) \\
\hline 10.11.2007 19:22:47 & 29784 & 171 & I2 & Ascending & VV & $19-26$ & Sea ice and ocean (Fram Strait) \\
\hline 31.01.2009 14:42:33 & 36194 & 68 & $\mathrm{I} 2$ & Descending & $\mathrm{HH}$ & $19-26$ & Sea ice (Greenland coast) \\
\hline
\end{tabular}

\section{REsults}

This section presents results after applying the technique described in the previous section to scenes covering the Fram Strait. The scenes (listed in Table I) were selected because they contain sufficient land enabling proper calibration of the Doppler shifts as described in section III-C. The examples show estimates with an approximate grid spacing of $1 \mathrm{~km} \times$ $1 \mathrm{~km}$, i.e. the axes can be interpreted both as indices in the RVL grid and as approximate length in kilometers.

Figure 5a shows a scene acquired 31 January 2009 (descending pass, $\mathrm{HH}$ polarization). The intensity image (figure $5 b$ ) shows a solid ice cover with some small leads off the North-East Greenland coast and some distinct ice floes in open water in the fjords of Greenland (top of the image). If we compare azimuth lines $0-160$ to lines $160-300$ in the radial velocity image (figure 5c) we see a significant discontinuity in the flow field. Figure 5e shows the profile obtained by averaging the RVL measurements (figure $5 \mathrm{c}$ ) over range. There is a band of higher estimated speeds in the middle of the scene showing a flow of ice moving at an average of $44 \mathrm{~cm} / \mathrm{s}$ South-East towards the Fram Strait (see figure 5a and note that negative values denote drift towards the antenna). Because we are measuring only one component of the drift, the SouthEast drift direction is a best guess based on the sign of the radial surface velocity and the fact that the ice movement is restricted by land on one side. The Doppler standard deviation in this area is $2.85 \mathrm{~Hz}$ which corresponds to a standard deviation in the ground range radial velocity of $18.4-24.7 \mathrm{~cm} / \mathrm{s}$ depending on the incident angle (see equation (7)). From the intensity image it is reasonable to assume that the solid and homogeneous piece of ice on lines 300-350 on the left hand side of figure $5 \mathrm{~b}$ is fast ice. Over this region, the estimated radial velocity is mostly zero indicating that the calibration is reasonable. The standard deviation image shown in figure $5 \mathrm{~d}$ has low values over land (in the order of $3 \mathrm{~Hz}$ ), so we expect good calibration of the Doppler anomalies. Visually, the ice floes in the fjord seem to be free floating, yet the radial velocity indicates no movement. It is important to remember that this means no motion in the radial direction, i.e. the floes may still have a velocity component along the azimuth direction which is not observable using this method. Also, note that leads in the ice are easily distinguished in the Doppler standard deviation image. This is most likely due to mixed content within the estimation cell as discussed in section III-E. The radial surface velocity plot also shows that the removal of the periodic bias was successful. Although the method described in section III-B2 works for scenes with reasonably small variation in Doppler across the scene, the amplitude can sometimes be difficult to estimate, especially in scenes with low signal-to-noise ratio. We believe it is possible to further reduce $f_{\text {bias }}$ by compensating the periodic modulation at the raw data level. This will be a topic for future work.

Figure 6 shows the position of two scenes separated by three days covering the entire Fram Strait with land both on the Greenland side and on the Svalbard side. The RVL measurements for the scenes are presented in figure 7 and figure 8 . Since the scenes are relatively close in time it was possible to run traditional cross-correlation tracking between the scenes. We first present results using our method and then compare with the cross-correlation drift as well as measurements by a drifting ice buoy (also shown in figure 6)

Figure 7 is an ascending pass acquired on 7 November 2007. In figure $7 \mathrm{~b}$ we see a solid sea ice cover in the top half of the image and open water in the bottom half. The estimated RVL product is shown in figure 7c. Over the ice, a band of higher drift speed can be observed (azimuth cells 800-1000). An average speed profile over range is shown in figure 7e, showing a clear difference in drift speed between azimuth lines $800-1000$ and lines 1000-1200. Note that the measured surface velocities over ocean are much higher over the water than over the ice (which is to be expected) and have therefore been truncated at $60 \mathrm{~cm} / \mathrm{s}$ to better see the dynamic range of the ice speeds. The steep topography on the Svalbard side prevents us from estimating the Doppler over land with good precision as indicated by the high standard deviation (figure 7d). The standard deviation on the Greenland side is lower, however the number of cells with a standard deviation less than $5 \mathrm{~Hz}$ were limited. Thus, there is likely an uncertainty of a few $\mathrm{Hz}$ in the absolute calibration as well.

Figure 8 is an ascending pass acquired on 10 November 2007. Figure $8 \mathrm{~b}$ looks similar to figure $7 \mathrm{~b}$ with comparable ice cover. However, a change in the ice edge as well as several big leads can be observed. Figure $8 \mathrm{c}$ shows a similar but wider band with comparable drift speed to that in figure $7 \mathrm{c}$, with a strong drift towards the South-West between azimuth lines 600 and 800. It is well known that on average, the Fram Strait has a southward outflow of ice as shown in figure 1. The drift in the center of both figures $7 \mathrm{c}$ and $8 \mathrm{c}$ is consistent with this trend. An average profile over all RVL range cells is shown in figure 8e. Again, the profile shows a significant difference between the estimated speed (compare azimuth lines 600-800 to lines 800-1000). The Doppler standard deviation over land in figure $8 \mathrm{~d}$ is high on the Svalbard side and lower on the Greenland side. This is also consistent with figure $7 \mathrm{~d}$.

Since we are measuring the velocity along the pointing direction of the antenna, there will undoubtedly be a contribution from both the vertical motion of the ocean and the horizontal movement of the ice. It is known that ocean waves may penetrate far into the ice cover [22]. Both scenes show strong 
wave patterns in the marginal ice zone (especially the scene from Nov. 7). By visual inspection we found that the waves seem to subside before reaching the band of higher velocities which is observable in figure $7 \mathrm{c}$. If the measurements were severely affected by swell we would expect more variation in the estimated Doppler close to the ice edge. The extent to which swells influence the measurements is unknown and needs further investigation.

Because the two scenes were three days apart it was possible to obtain a two-dimensional drift field using traditional crosscorrelation tracking between the scenes. We used a recent cross-correlation tracking method by Komarov and Barber [1] to estimate the 2-D velocity field from the two images. The algorithm is a variation of the traditional pyramid correlation motion estimation scheme (see e.g. [23]) which includes rotation estimation. For the correlation analysis, the two raw data (Level-0) scenes were focused to SLC, this time including window functions to reduce ghosting effects, and subsequently geocoded to a Universal Transverse Mercator (UTM) grid with a $20 \mathrm{~m}$ pixel spacing. The 2-D drift field obtained from the correlation analysis has a large ice field moving along the azimuth direction (figure 9a) and one field moving almost directly along the range direction (figure 9b). Panel (a) covers lines 830-1000 in the RVL image (c), while panel (b) covers lines 570-800. If we project the derived displacement vectors onto the range direction of the scene from 10 Nov. 2007 we get figure $9 \mathrm{c}$ which shows a trend similar to figure $8 \mathrm{c}$ (shown again as figure $9 \mathrm{~d}$ for comparison).

In general, it is not possible to compare such different measurements directly. Since the RVL measures a component of the instantaneous drift speed it could, in principle, have shown a drift in the opposite direction of the cross-correlation drift if the drift direction had turned. Therefore, we present this figure as an interesting coincidence and as a qualitative indicator that the two regions we see in the RVL image correspond to real geographical differences in the drift direction.

For the upper part, near Greenland the estimated drift had a mean of $5 \mathrm{~cm} / \mathrm{s}$ (figure $9 \mathrm{a}$ ), whereas the lower region had a mean speed of $16 \mathrm{~cm} / \mathrm{s}$ (figure $9 \mathrm{~b}$ ). The maximum speed measured by the cross-correlation method was $19.6 \mathrm{~cm} / \mathrm{s}$. When projected along the line-of-sight, this corresponded to a speed of $17.6 \mathrm{~cm} / \mathrm{s}$. The Doppler method estimates the largest drift speeds at $40-60 \mathrm{~cm} / \mathrm{s}$ (see figure $8 \mathrm{e}$ ), which is considerably higher.

Note that there is a large gap over the ice in the projected cross-correlation drift field (range columns $0-45$ of figure 9). Over this region, ice that was visible in the first image had moved out of the second image and the algorithm was therefore unable to match patterns across the two scenes. In this respect our method has a clear advantage as we are able to estimate the ice drift over the whole scene (although the measured component may be zero).

For a direct comparison, the most relevant sensor to compare to is a buoy with high time resolution. Through the International Arctic Buoy Programme we were able to obtain buoy measurements from a single ARGOS buoy (ID number 35235) which overlapped with the two scenes from 2007 and has a time resolution of less than an hour. The buoy is within the

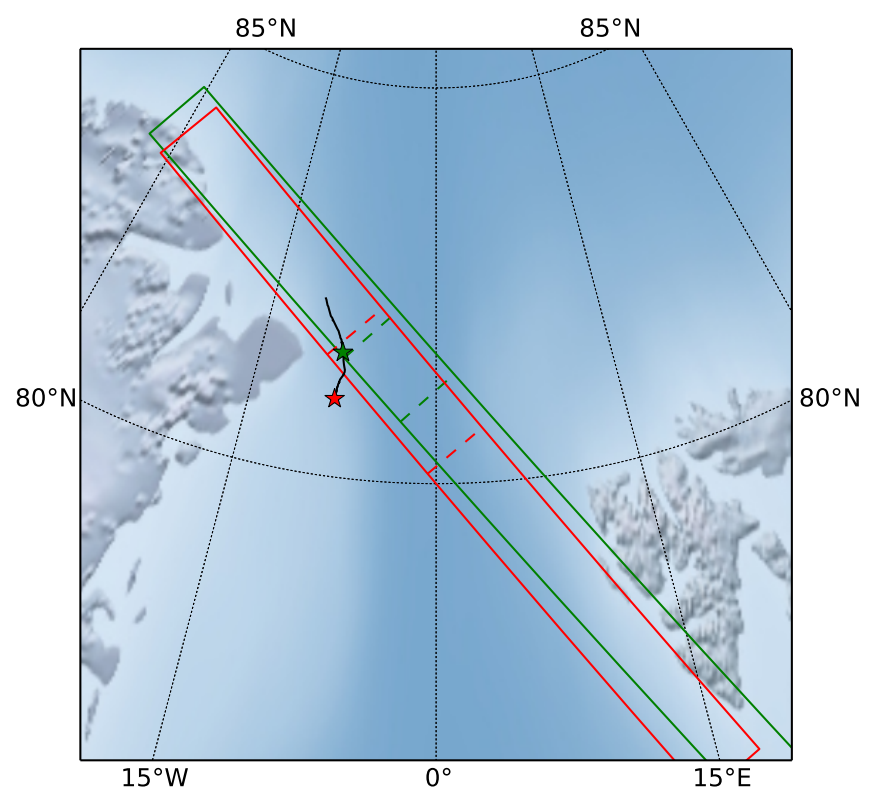

Fig. 6. Buoy drift from 6 November to 11 November. Scene footprints and nearest (in time) buoy position are indicated on the 7. November (green) and 10. November 2007 (red). The extent of the bands of higher drift speed observed in figure 7 and 8 is shown for each scene with dashed lines.

scene from 7 November 2007 (marked by a star in figure 7a and $7 \mathrm{~b}$ ), and just outside the scene from 10 November 2007 (see figure 8a). Drift speeds were obtained by first order differentiation of the buoy positions (from Doppler tracking, not GPS) with location class 3 (estimated radius of error less than $250 \mathrm{~m}$ ). From 19:00 to 20:00 UTC on 7 Nov. 2007 (the scene was acquired at 19:16), the drift speed varied from 0.4 to $1.3 \mathrm{~m} / \mathrm{s}$, which is much higher than the estimated correlation measurements. This is to be expected as the cross-correlation drift averages motion over three days. The projection of the 40 $\mathrm{cm} / \mathrm{s}$ buoy speed onto the line of sight gave a radial drift of 26 $\mathrm{cm} / \mathrm{s}$. Due to the uncertainty in the ARGOS buoy position, the buoy could potentially be in one of several RVL cells. Over the surrounding RVL cells, radial velocities were measured at between 26 and $58 \mathrm{~cm} / \mathrm{s}$.

For the 10 November scene, the buoy was drifting almost directly along the pointing direction with a projected speed of $22 \mathrm{~cm} / \mathrm{s}$. The closes RVL cells show radial speeds of 36 $\mathrm{cm} / \mathrm{s}$ up to $94 \mathrm{~cm} / \mathrm{s}$ with model standard deviations around $8 \mathrm{~cm} / \mathrm{s}$. However, there is much uncertainty involved in this comparison because the buoy is well outside the scene and also the buoy position was ambiguous (there were multiple positions for the same time stamp, both of which were marked as good quality estimates).

The measurements by the Doppler RVL method, the buoy and the cross-correlation all show the same trend with movement first parallel to the azimuth direction, then along the radial direction.

Buoy measurements provide good estimates of the drift speed, but only provide point measurements. It is therefore difficult to say something about how representative the bouy measurement is, i.e. the geographical extent showing the same drift characteristics. Our single buoy measurement helps to 
illustrate this point as it is very close to the discontinuity between two large areas of distinctly different drift. We hope that the combination of the RVL measurements with other sources of drift information, both from buoys and from correlation tracking, may help to improve future drift models. In particular, short term forecasting of the drift direction may benefit from the combined drift history obtained through correlation tracking with the instantaneous RVL measurements. However, the model would have to account for only observing the radial component.

\section{CONClusion}

Using ASAR data, we have demonstrated the Sentinel-1 Level-2 RVL product in the context of tracking Arctic sea ice drift, focusing on Fram Strait. Our results show that it is possible to map discontinuities which may indicate shear in the ice and detect extended areas with similar drift characteristics. We obtain higher resolution and better precision than what has been possible with the ASAR WSM Doppler grid. Although we are not able to estimate 2-D drift fields using the Doppler method, we have shown that when the radar pointing direction aligns with the ice drift we can observe higher speeds than what is possible using time series methods. The estimated radial velocity field is believed to be most useful in combination with existing time series methods and buoy measurements.

With the launch of Sentinel-1 the availability of the RVL product will be easily available and may provide additional insight on ice drift when combined with traditional correlation tracking. The Sentinel-1 platform will also have better attitude steering and antenna monitoring than Envisat, which will provide very precise measurements of the ice motion, forming a good basis for further validation of the method.

\section{ACKNOWLEDGMENTS}

The authors would like to thank Geir Engen (NORUT) for providing the Doppler estimation and side-band correction routines and Yngvar Larsen (NORUT) for providing the GSAR SAR processing software. Also, we would like to thank the reviewers for encouraging and insightful feedback which increased the quality of the paper. Ignatius Rigor with the Applied Physics Laboratory, University of Washington was kind enough to provide the buoy measurements. Envisat data are copyright European Space Agency.

\section{REFERENCES}

[1] A. S. Komarov and D. G. Barber, "Sea ice motion tracking from sequential dual-polarization RADARSAT-2 images," IEEE Transactions on Geoscience and Remote Sensing, vol. 52, no. 1, pp. 121-136, Jan. 2014.

[2] M. Haller, B. Brümmer, and G. Müller, "Atmosphereice forcing in the transpolar drift stream: results from the DAMOCLES ice-buoy campaigns 20072009," The Cryosphere, vol. 8, no. 1, pp. 275-288, Feb. 2014.

[3] M. Leppäranta, The drift of sea ice. Springer, 2005.

[4] R. Romeiser, H. Runge, S. Suchandt, R. Kahle, C. Rossi, and P. S. Bell, "Quality Assessment of Surface Current Fields From TerraSAR$\mathrm{X}$ and TanDEM-X Along-Track Interferometry and Doppler Centroid Analysis," IEEE Transactions on Geoscience and Remote Sensing, vol. PP, no. 99, pp. 1-14, 2013.

[5] D. N. Thomas and G. S. Dickmann, Eds., Sea ice, 2nd ed. WileyBlackwell, 2009.
[6] M. Aulard-Macler, "Sentinel-1 Product Definition S1-RS-MDA-527440," MacDonald, Dettwiler and Associates Ltd., Tech. Rep., 2011.

[7] M. J. Rouault, A. Mouche, F. Collard, J. a. Johannessen, and B. Chapron, "Mapping the Agulhas Current from space: An assessment of ASAR surface current velocities," Journal of Geophysical Research, vol. 115, no. C10, p. C10026, Oct. 2010.

[8] A. A. Mouche, F. Collard, B. Chapron, K.-F. Dagestad, G. Guitton, J. A. Johannessen, V. Kerbaol, and M. W. Hansen, "On the Use of Doppler Shift for Sea Surface Wind Retrieval From SAR," IEEE Transactions on Geoscience and Remote Sensing, vol. 50, no. 7, pp. 2901-2909, Jul. 2012.

[9] B. Chapron, F. Collard, and F. Ardhuin, "Direct measurements of ocean surface velocity from space: Interpretation and validation," Journal of Geophysical Research, vol. 110, no. C7, pp. 1-17, 2005.

[10] Y. Fujiyoshi, K. Osumi, M. Ohi, and Y. Yamada, "Sea Ice Identification and Derivation of Its Velocity Field by X-Band Doppler Radar," Journal of Atmospheric and Oceanic Technology, vol. 30, no. 6, pp. 1240-1249, Jun. 2013.

[11] M. W. Hansen, K. Kloster, K.-F. Dagestad, S. Sandven, and J. A. Johannesen, "Retrieval of sea ice drift from SAR Doppler shift," in Proc. ESA Living Planet Symposium (SP-709). Bergen: ESA, 2010, pp. $1-5$.

[12] G. Engen and H. Johnsen, "High-precision Doppler frequency estimation for ocean applications," in Proc. SEASAR (SP-709). Tromsø: ESA, 2012, pp. $1-4$.

[13] —, "Sentinel-1 Doppler and ocean radial velocity (RVL) algorithm definition," NORUT, Tech. Rep., 2010. [Online]. Available: https://sentinel.esa.int/c/document_library/get_file? \&folderId $=349449$ \&name $=$ DLFE-5009.pdf

[14] I. G. Cumming and F. H. Wong, Digital processing of synthetic aperture radar data: algorithms and implementations. Artech House, 2005.

[15] R. Bamler, "Doppler Frequency Estimation and the Cramér-Rao Bound," IEEE Transactions on Geoscience and Remote Sensing, vol. 29, no. 3, pp. 385-390, 1991.

[16] M. W. Hansen, F. Collard, K.-F. Dagestad, J. a. Johannessen, P. Fabry, and B. Chapron, "Retrieval of sea surface range velocities from Envisat ASAR Doppler centroid measurements," IEEE Transactions on Geoscience and Remote Sensing, vol. 49, no. 10, pp. 3582-3592, Oct. 2011.

[17] R. K. Raney, "Doppler properties of radars in circular orbits," International Journal of Remote Sensing, vol. 7, no. 9, pp. 1153-1162, 1986. [Online]. Available: http://www.tandfonline.com/doi/abs/10.1080/ 01431168608948916

[18] A. Recchia, A. M. Guarnieri, N. Miranda, F. Collard, D. D. Aria, D. Giudici, P. Milano, A. Monti Guarnieri, and D. D'Aria, "Impact of the antenna stability on the Doppler centroid frequency," Proc. IGARSS, pp. 1516-1519, Jul. 2011.

[19] L. L. Scharf, Statistical Signal Processing, Detection, Estimation, and Time Series Analysis. Addison Wesley, 1991.

[20] S. Nghiem and S. Martin, "A laboratory study of the effect of frost flowers on C band radar backscatter from sea ice," Journal of Geophysical Research, vol. 102, no. C2, pp. 3357-3370, 1997.

[21] G. Sotis, "DORIS products specifications," Tech. Rep., 2008.

[22] A. L. Kohout, M. J. M. Williams, S. M. Dean, and M. H. Meylan, "Storm-induced sea ice breakup and the implications for ice extent," Nature, vol. 509, no. 7502, pp. 604-607, 2014.

[23] M. V. Thomas, C. Kambhamettu, and C. A. Geiger, "Motion tracking of discontinuous sea ice," IEEE Transactions on Geoscience and Remote Sensing, vol. 49, no. 12, pp. 5064-5079, Dec. 2011. 


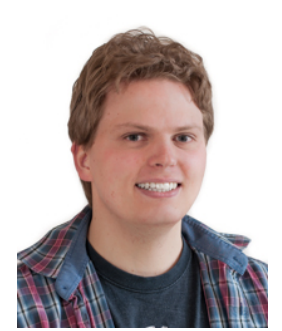

Thomas Kræmer received the M.Sc. degree in data analysis and sensor technology in 2011 from the University of Troms $\varnothing$, focusing on segmentation of sea ice using polarimetric synthetic aperture radar (SAR) images. He is currently pursuing the Ph.D. degree with the Barents Remote Sensing School (BARESS) at UiT - The Arctic University of Norway, Troms $\varnothing$, Norway. His current research interests are on algorithms for automated analysis of SAR images for sea ice applications.

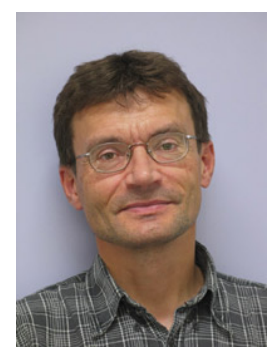

Harald Johnsen received his M.Sc. degree in 1981 and his Ph.D. in 1984 from the University of Troms $\varnothing$. His scientific experiences are within plasma physics, signal analysis and remote sensing. After the graduation, he worked two years at the Auroral Observatory in Troms $\varnothing$ before he joined Norut in 1986 as a research scientist within the Earth Observation Group. From 1994 to 2002 he was the Research Manager at the same institution with responsibility for the remote sensing activities. At present he is a senior researcher at Norut and an adjunct professor at University of Troms $\varnothing$. Dr. Johnsen's main research interest at present is within SAR ocean and sea ice applications and SAR interferometry applications.

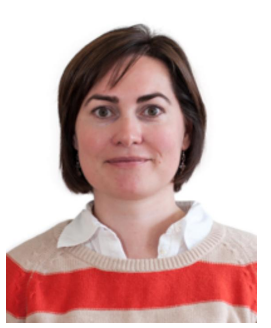

Camilla Brekke (M'12) received the Cand. Scient. and Ph.D. degrees from Department of Informatics, University of Oslo, Norway, in 2001 and 2008, respectively. In 2009, she joined the Department of Physics and Technology at UiT - The Arctic University of Norway as an Associate Professor. She is with the Earth Observation Laboratory and the Barents Remote Sensing School. Her current research interests cover signal and image processing of synthetic aperture radar (SAR) data for arctic and marine applications. In 2001, she was a System Developer with Ericsson, Asker, Norway. From 2001 to 2002, she was a Young Graduate Trainee with the European Space Agency (ESA), Noordwijk, Netherlands. From 2002 to 2009, she was a Scientist with the Norwegian Defense Research Establishment (FFI), Kjeller, Norway. From October 2012 to January 2013, she was a Visiting Scientist with the Jet Propulsion Laboratory, Pasadena, CA, USA. 


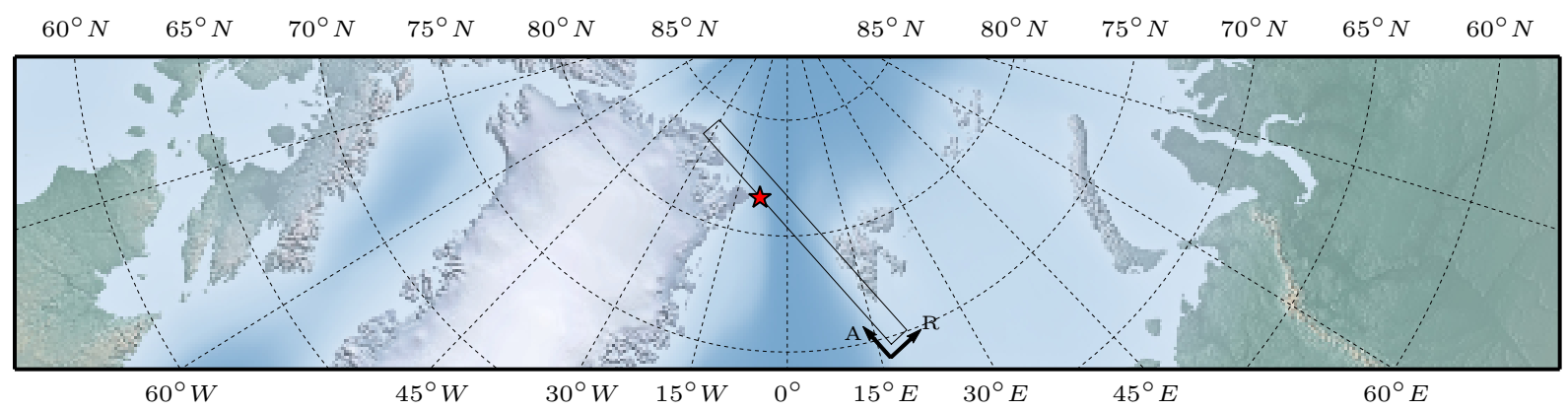

(a)

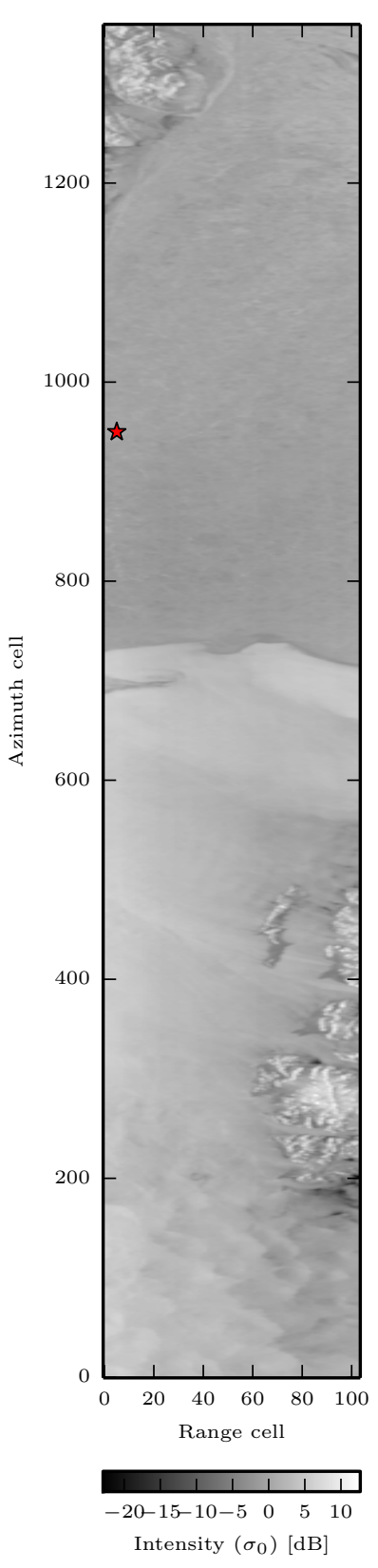

(b)

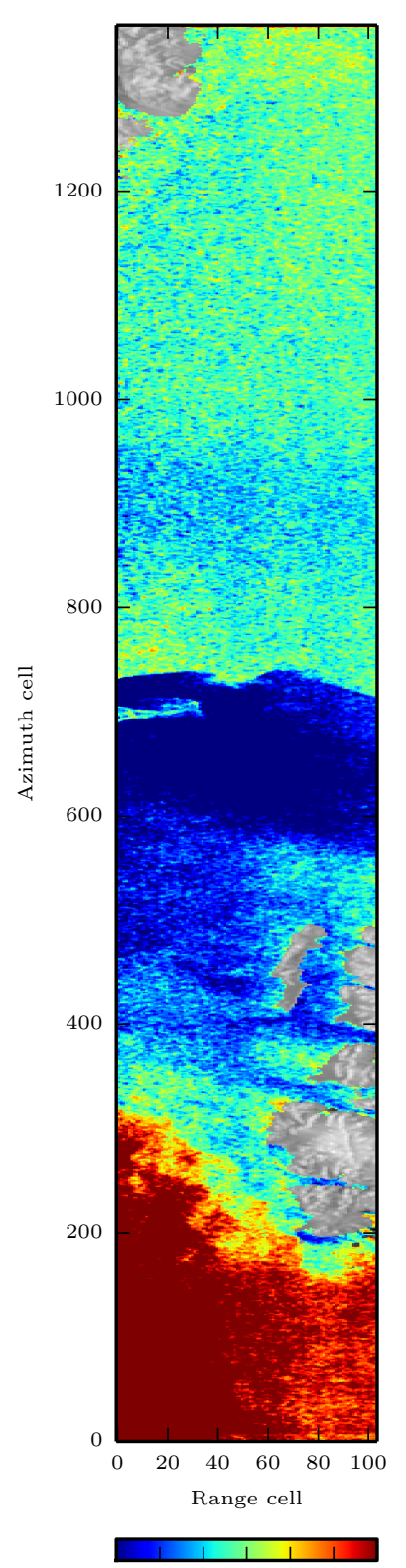

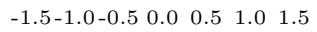

Radial surface velocity $[\mathrm{m} / \mathrm{s}]$

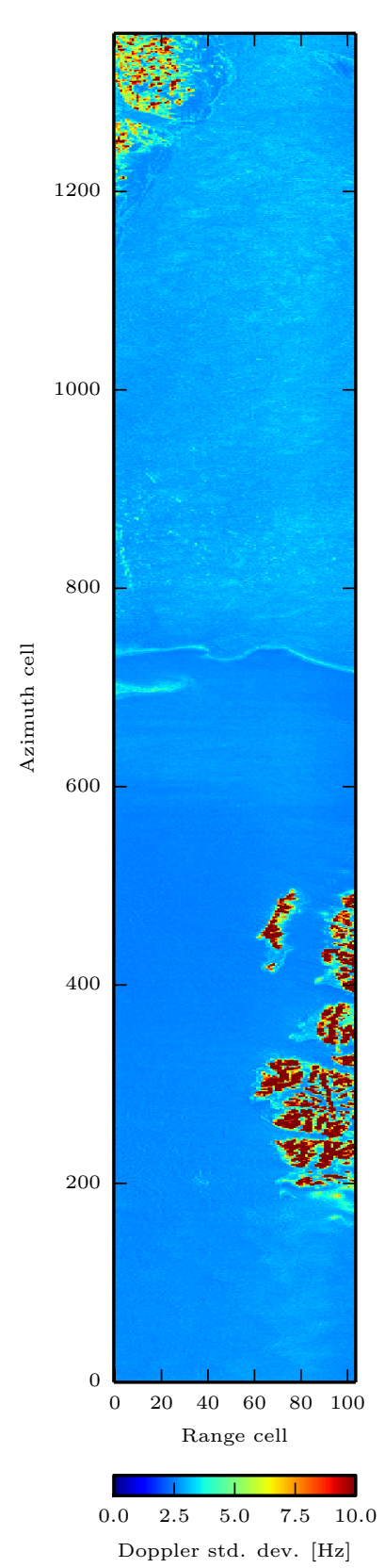

(d)

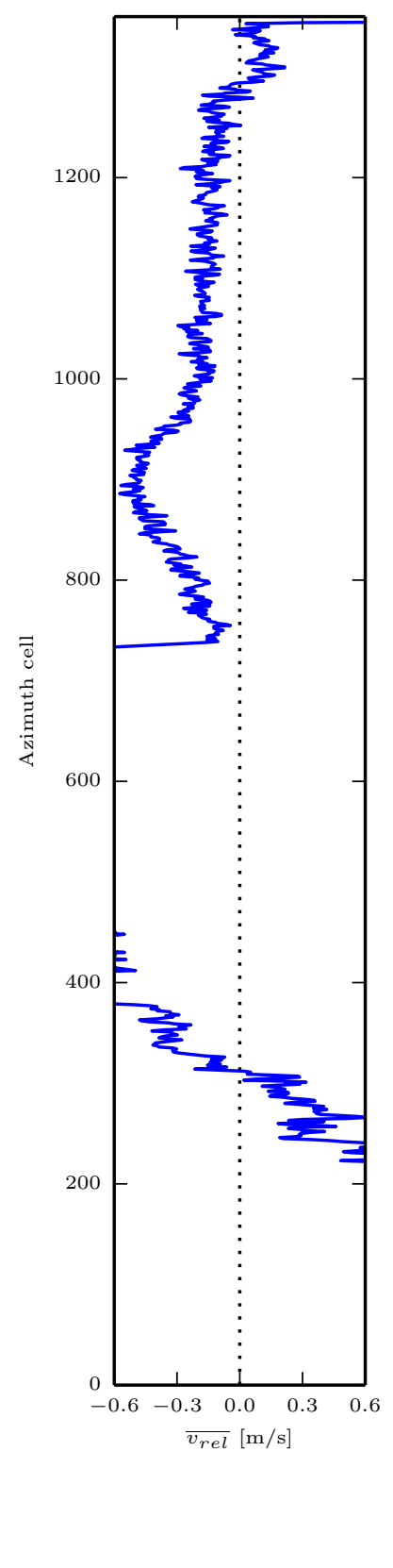

(e)

Fig. 7. Scene from 7 November 2007 (ascending pass, VV polarization) with almost no radial motion along the Greenland coast, but a strong band of higher motion in the middle of Fram Strait. (a) Geographical position of the scene. The azimuth (A) and range (R) directions are indicated by arrows. (b) $\sigma_{0}$ intensity image. (c) Estimated ground range radial surface velocities. Note the band of higher speeds at lines $800-1000$ indicating a strong flow of ice towards the South-West. (d) Standard deviations of Doppler frequency measurements. (e) Average velocity profile. 


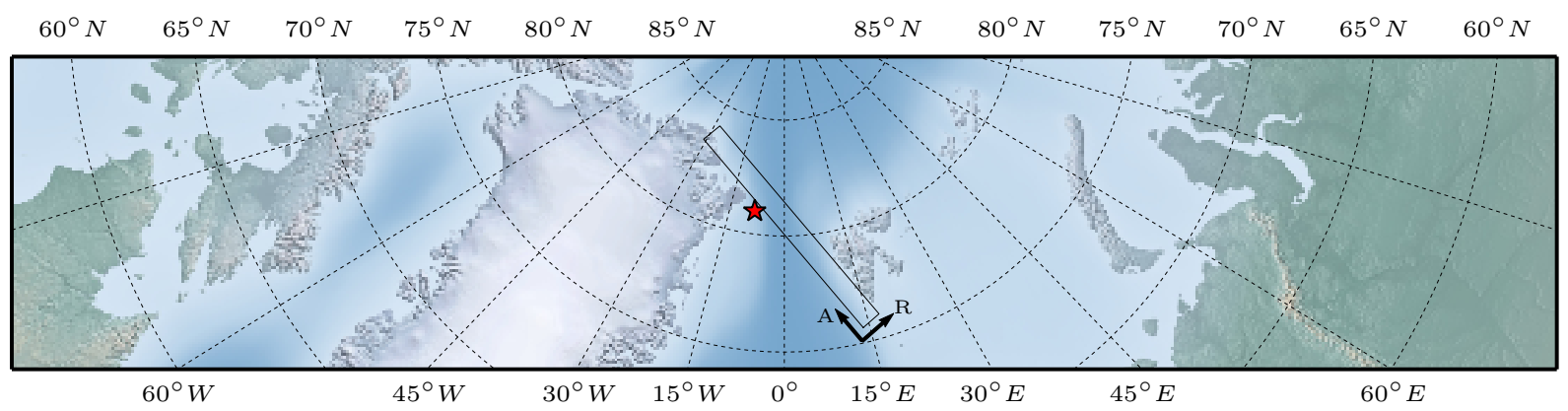

(a)

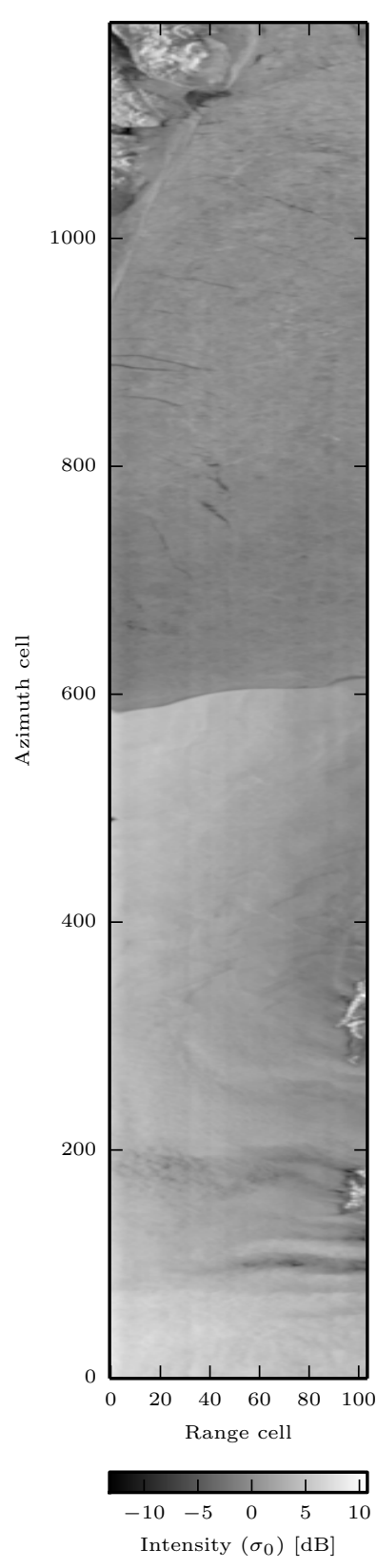

(b)

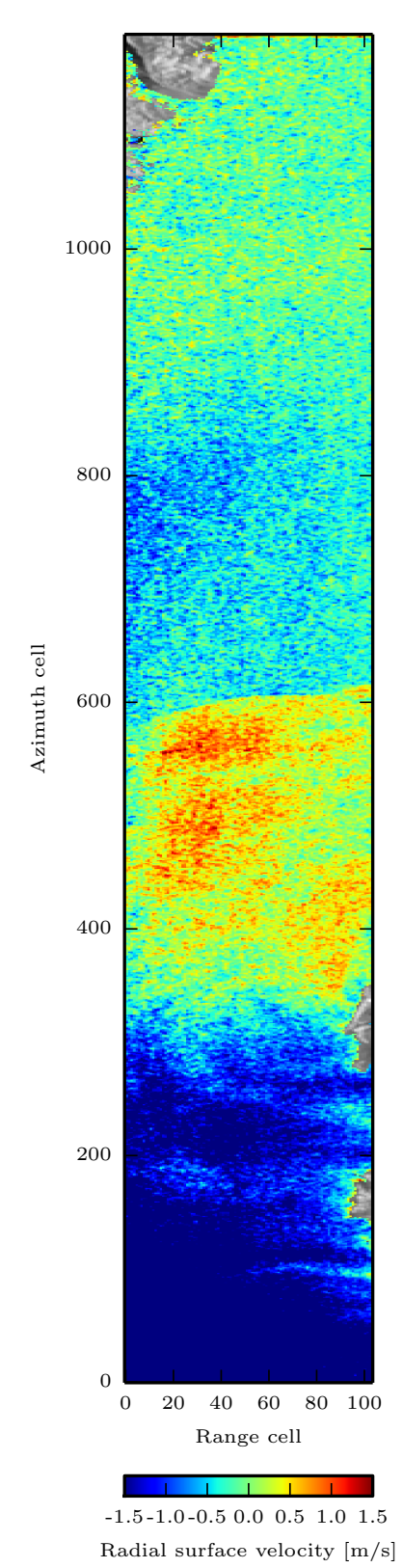

(c)

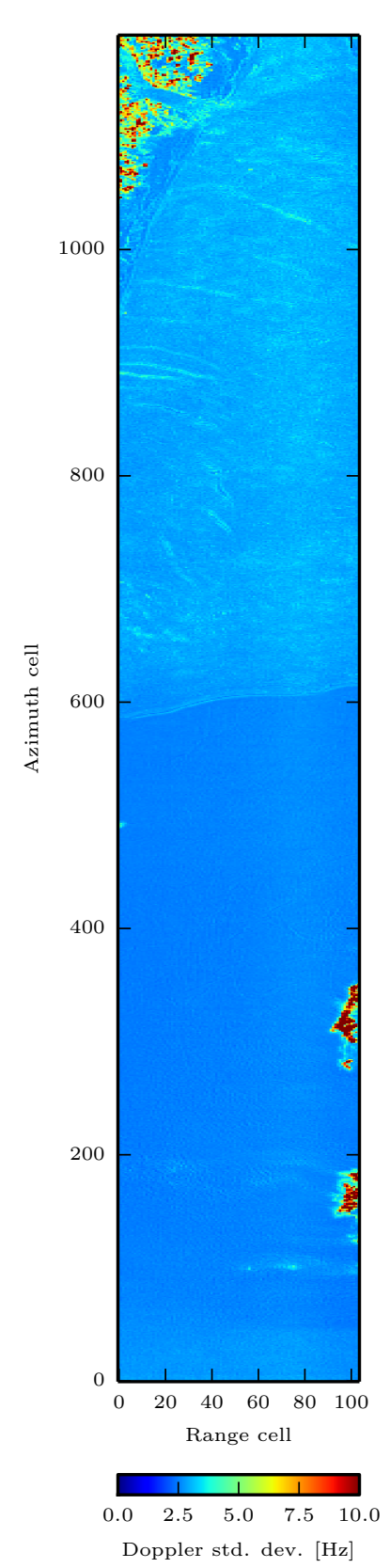

(d)

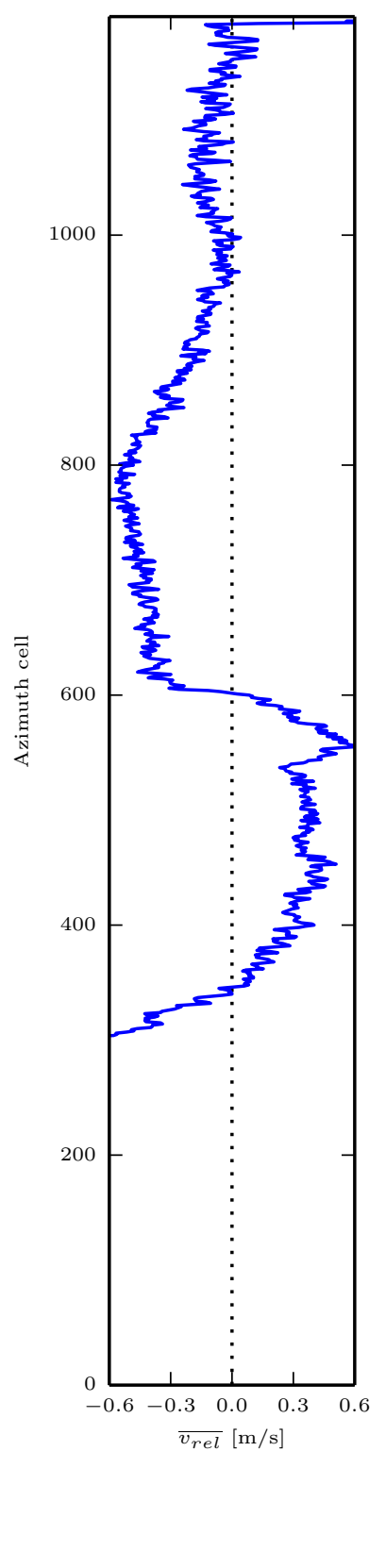

(e)

Fig. 8. Scene from 10 November 2007 (ascending pass, VV polarization) showing a wide band of higher radial motion in the middle of Fram Strait. (a) Geographical position of the scene. The azimuth (A) and range (R) directions are indicated by arrows. (b) $\sigma_{0}$ intensity image. (c) Estimated ground range radial surface velocities. Note the band of higher speeds at lines 600-800 indicating a strong flow of ice towards the South-West. (d) Standard deviations of Doppler frequency measurements over the pixels within each Doppler cell. (e) Average velocity profile. 


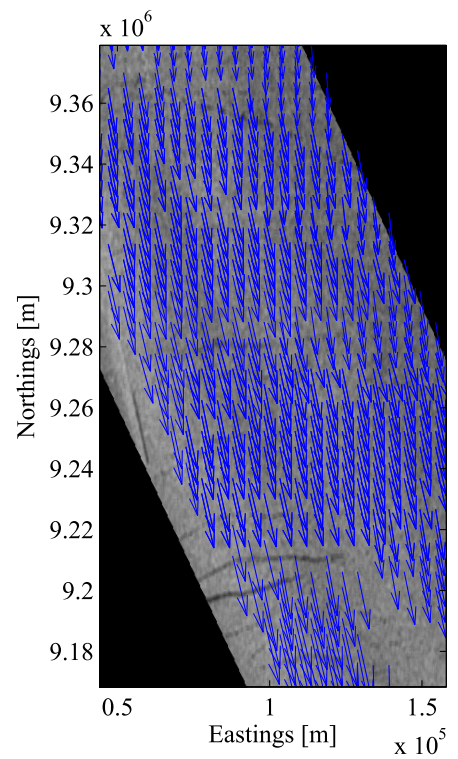

(a)

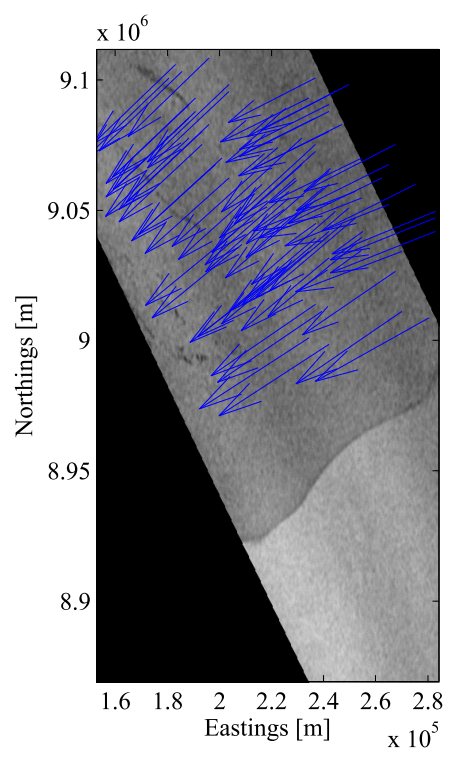

(b)

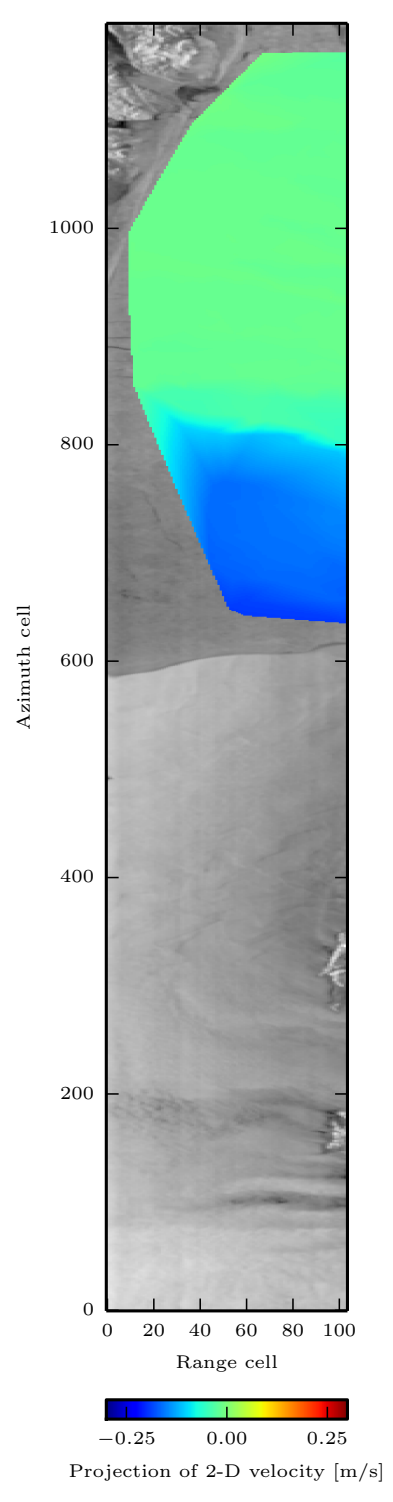

(c)

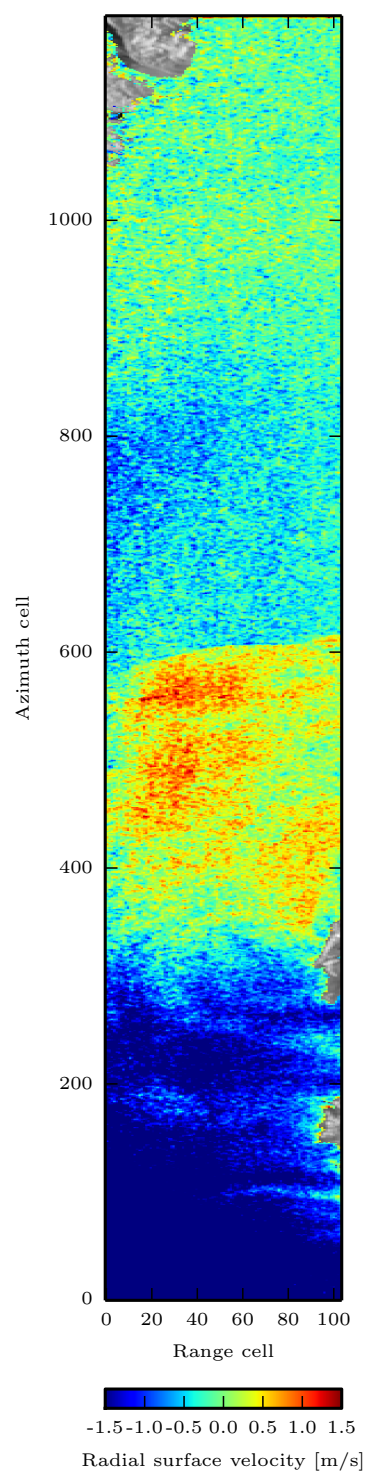

(d)

Fig. 9. Comparison of the output from the cross-correlation ice tracking with the estimated radial velocities for the two overlapping scenes shown in figure 7 and figure 8. (a) and (b) 2-D drift vectors derived using cross-correlation plotted over the latter scene in the UTM projection. The first shows dominant ice drift parallel to the azimuth direction. The second shows dominant ice drift parallel to the range direction. (c) The projection of the cross-correlation drift field onto the range direction of figure 8 . Two distinct regions of different ice drift can be observed, consistent with (a) and (b). (d) The radial surface velocity in figure $8 \mathrm{c}$ shown again to make the comparison easier. 